\title{
Tangerding, Clemens Maria, Der Drang zum Staat. Lebenswelten in Würzburg zwischen 1795 und 1815
}

\section{Guillaume Garner}

\section{OpenEdition}

\section{Journals}

Édition électronique

URL : http://journals.openedition.org/ifha/6522

DOI : $10.4000 /$ ifha.6522

ISSN : 2198-8943

Éditeur

IFRA - Institut franco-allemand (sciences historiques et sociales)

Référence électronique

Guillaume Garner, «Tangerding, Clemens Maria, Der Drang zum Staat. Lebenswelten in Würzburg zwischen 1795 und 1815 », Revue de l'IFHA [En ligne], Date de recension, mis en ligne le 01 janvier 2012, consulté le 22 septembre 2020. URL : http://journals.openedition.org/ifha/6522 ; DOI : https://doi.org/ $10.4000 /$ ifha. 6522

Ce document a été généré automatiquement le 22 septembre 2020.

(C)IFHA 


\title{
Tangerding, Clemens Maria, Der Drang zum Staat. Lebenswelten in Würzburg zwischen 1795 und 1815
}

\author{
Guillaume Garner
}

1 Issu d'une thèse soutenue en cotutelle dans les universités de Dresde et de Paris VIII, cette monographie se donne pour objectif d'appréhender les bouleversements de la période révolutionnaire et impériale (les années 1795-1815) dans une ville, Wurtzbourg, qui a connu pas moins de quatre appartenances politiques successives en deux décennies. C.M.T. ne propose cependant pas une histoire politique de facture traditionnelle, mais fonde son étude sur la notion de «monde vécu » (Lebenswelt) forgé par Alfred Schütz et sur celle d'« importance » (Relevanz) pour cerner l'impact que les événements des deux décennies étudiées ont pu avoir - ou non - sur les habitants de Wurtzbourg. L'ouvrage s'efforce donc de reconstituer le "monde vécu » de cinq catégories sociales - les professeurs d'université, les hauts-administrateurs, les artistes, la petite et moyenne bourgeoisie et les voyageurs - à chacune desquelles est consacré un chapitre. Comme l'auteur le précise en introduction, cette approche centrée sur les acteurs conditionne le choix de sources qui comprennent des correspondances épistolaires, des suppliques et pétitions, des lettres de voyages, des journaux intimes. Ce faisant, C.M.T. entend apporter des éléments de réponse à des questions selon lui négligées par l'historiographie : quels liens peut-on établir entre l'activité professionnelle et éditoriale d'universitaires ou les créations et la situation personnelle (professionnelle et financière) des professeurs d'université ou des artistes ? En quoi l'approche par la notion de "monde vécu » permet-elle de jauger le degré de politisation des populations urbaines?

2 Comme l'exprime le titre de cet ouvrage, l'ensemble des groupes et des acteurs étudiés partage un besoin d'État, plus exactement la recherche d'un lien étroit avec l'État et les autorités politiques en place. Le deuxième chapitre souligne en particulier à quel point, dans un contexte de grande incertitude politique et institutionnelle, les professeurs de l'université de Wurtzbourg cherchent par leurs publications et leurs écrits à préserver 
un statut social et professionnel menacé, le cas échéant en multipliant les critiques acerbes contre leurs collègues. Loin de chercher à se situer dans une sphère séparée de l'État pour y consolider leur autonomie intellectuelle et professionnelle, les savants universitaires cherchent au contraire à entrer au service de l'État, à se profiler comme des serviteurs fiables de ce dernier. Mutatis mutandis, les mêmes conclusions sont formulées à propos des administrateurs et des artistes, même si dans ces deux derniers cas les sources disponibles sont moins abondantes. La recherche d'une relation privilégiée à l'État caractérise également la petite et moyenne bourgeoisie urbaine, à l'exemple des cochers, dont l'activité est fortement dépendante en temps de guerre de la demande publique, et qui n'ont de cesse de demander la création d'une corporation pour contenir la concurrence d'autres acteurs. Quant à la bourgeoisie urbaine, elle proteste avant tout contre l'établissement de la conscription militaire générale en 1804, sa demande du rétablissement du privilège urbain en ce domaine étant vaine. Les lettres de voyages enfin, en occultant les bouleversements ayant affecté la ville et en soulignant les éléments de continuité, exprimeraient selon C.M.T. un besoin de structures politiques stables et valoriseraient ainsi la figure du prince comme facteur de cette stabilité, comme «bon pasteur ».

3 C.M.T. souligne ainsi que derrière la diversité des réactions des différents acteurs étudiés apparaît une constante : la recherche de la protection par l'État. Il s'agit cependant d'une recherche apolitique, la politisation de la société urbaine n'étant selon lui possible qu'après 1815 , une fois la ville entrée dans une période de stabilité politique et institutionnelle.

4 Guillaume Garner (ENS Lyon) 